\title{
Science: Scarcity Versus Plenty
}

\author{
Almada $F^{1 *}$, Fernando $C^{2}$ and Vicente $A^{3}$ \\ ${ }^{1}$ Retired University Professor / Independent Researcher, Portugal \\ ${ }^{2}$ University of Madeira - CITUR, Portugal \\ ${ }^{3}$ University of Beira Interior - CIDESD, Portugal
}

*Corresponding author: Almada F, Retired University Professor / Independent Researcher, Portugal

\begin{tabular}{|c|c|}
\hline ARTICLE INFO & ABSTRACT \\
\hline Received: 幽 July 08, 2021 & $\begin{array}{l}\text { Citation: Almada F, Fernando C, Vicente A. Science: Scarcity Versus Plenty. Biomed J Sci } \\
\text { \& Tech Res 37(3)-2021. BJSTR. MS.ID.005995. }\end{array}$ \\
\hline
\end{tabular}

\section{Introduction}

Managing in a context of scarcity is radically (and can go up to the level of rupture, in the kuhnian sense of the term) different from managing in a world of plenty.

In order not to stick to the slogan, we are concise and:

1. Of the Objectives: scarcity imposes the objectives on us within the necessary (sometimes only the essential, the basic); in the context of plenty we must find, among the many possible, the desired objectives;

2. From the Strategy (which way): we have the adjustment of the possible paths (naturally tight) and the demand (or impositions) of the necessary resources versus avoiding waste and maintaining the focus on the action (or multiple actions) defined as desired, although, of course, within the available resources;

3. From the Tactic (where we seek to give body, to make reality, to the previous points): we have the economy of means in order not to waste resources so that we are not blocked by the faults versus the saving resources so that we do not have to reduce the target objectives or the quality with which each of them can be achieved;

4. Of Technology: we have the lowest possible costs, saving resources and restricting to the indispensable versus the investment in quality, knowing that technology has a reproductive sense and sometimes investing in routes that have no immediate utility allows saving, in the medium / long term, because there are solutions "in portfolio" that can be made available and that show alternatives that would not otherwise even be thought of (as well as, the demand carried out beyond the needs of the immediate allow identifying costs and collateral risks that, in this way, can be avoided);

5. From Operationalization: we are looking to meet the partial objectives in a parsimonious way, versus seeing how far we can go with the available resources, in search of the possible quality / quantity.

The basic structures, let's say "logistics", may even be identical in one case as in the other, but face very different challenges in how they have to combine the coherence and balances between the impositions and needs of the five points presented above.

A couple of examples:

A. In Education: the focus will be placed on the case of scarcity in points 3, 4 and 5 above presented, while in the case of abundancy, points 1 and 2 should be privileged, which open doors to the multiplicity of possible options;

B. In Research: the debate should focus on points 1 and 2, as there will be resources for the other points to materialize.

Let us leave science for a moment and look at what is happening in our daily life in trivial situations such as:

i. Feeding: in which we have "starved" versus "obesity". Two sick ways, but of contrary senses; 
ii. Clothing and other robes versus "unique clothing": with the need and indecision of choice, on the one hand and the difficulty of customizing on the other. For some time, men's "suit and tie" outfits were opposed to women's clothing that allowed other freedoms, but also concerns and costs. The uniforms, in addition to the sense of uniformity (uniform) also have this simplifying sense in the choice, being still an image of organization and structuring power by the image of "order", which they gave (gave for a while and in some contexts);

iii. Gadgets: which, when there is a shortage, are practically standardized, but abundance causes them to be used in a profuse way in which, often, the goals to be achieved are not even defined and, possibly, do not matter because the possession of a gadget is an end in itself;

iv. Toys: in which lack can restrict the richness of experiences, but can simultaneously stimulate imagination and wealth can foster curiosity, the tendency to seek novelty, but which can also lead to a superficiality in the exploitation in which it leads to an inconstancy and a "blasé" attitude in which everything loses the value (the values) that, in fact, it has;

v. Knowledge: that can be a tool, which is not an end in itself and is used in a parsimonious way. But can also be a mean of exhibition in which erudition is nothing more than a (sometimes even nozzle) way of projecting onto others of a verborrhea that in a position of power project onto their surroundings.

We are not defending any of these positions, not even the easy position to assume that a balance between them is necessary (which, to facilitate, we would still not need to try to define). We believe, however, that it is important to understand the alternatives of one position and another, to facilitate options and open visions of choice (which means preferring points 1 and 2, therefore defending the wealthy, within what we defend above). But, we think, plenty and scarcity have the same problems in the level of moving to operationalization, because it does not allow us to see, grasp, the possibilities that exist, since even if we can define desired objectives, we do not have the means to obtain them.

\section{In Science}

Today we have plenty of information and means to disseminate it. In other words, we have a situation of affluentity in which, however, the previous points 3,4 and 5 are still privileged. But we should have already started to focus on points 1 and 2 , focusing on the definition of objectives and strategies. We live, therefore, times of transition between the shortcomings that until recently (twenty or thirty years ago) we felt in which definitions are sought in coherence and balances between the five points indicated above, so that we can move from what Thomas Kuhn defined as "the crisis", to times of "normal science". If this passage takes place again, because the speed at which the changes (at the structural and deep level and not the mere incidents of the very current journey in the media and in social networks - and which we believe are simply swipe of the ambiguity in which we live at the level of the essential, the essential that was and the essentials that we need to be) are giving what may lead us to be permanently in crisis. However, the position we are defending is that the permanence of the crisis is a "passing thing", like always happens when we have not yet become accustomed to using new references and their consequences, the search for new balances and coherences.

We are living what we have pointed out in the "examples of our daily life", for food, toys, gadgets, etc.. The transition is not made progressively, but by rupture, in which the "world changes" and suddenly we find ourselves in a new framework, not even remembering what happened before. But in the framework of science, we have a "sui generis" product, as any researcher will recognize, although it often does not identify the specific characteristics that define that product. It is that knowledge, the product of science, is similar to a well-known Swiss cheese, the more product we have, more "holes" we have to manage. Since the function of science is advancing through the unknown, the questions from which we start lead us to more questions and even more questions, as it is visible in the conclusions of any research work (worthy of that name and not simply nicknamed in this way). Which puts us facing paradoxes, that we will give some examples:

\section{Rationality Versus Decision-Making Ability}

The "rational" (?) leads us to think that to decide, and decide well, we must have all the necessary information, have the time to work and then, calmly, make the decisions that are imposed. This is, however, the best way to make mistakes. Of making mistakes ... of all shapes and sizes. Because we will never have all the necessary information, neither the time to work all this information, nor the calm to decide when we try to solve really important things. Recently, this problem of incompleteness of the framework in which understanding of problems, decision-making and evaluation of the actions that are carried out and the results obtained has begun to gain weight (see, for example, the works of Herbert Simon).

At the root of the problems due to the need to act (in the most diverse institutional frameworks, from companies to states, from military structures to research organizations, from space agencies to large industrial organizations, from banks to planning institutions, that is, the most powerful institutions within which a mistake can be fatal at an economic level or even cause the death of many of its agents) without complete information on the issues on which they have to decide and make choices, obviously results from the limits we have set for any phenomenon, their borders, always being arbitrary (sometimes unconscious, even), leading 
to the need, in the best cases, to opt for a conditioned rationality (limited), which leads to decision-making being made considering: a) the time to decide; b) the relationship between the costs/benefits of obtaining more information; c) the lethality and importance of the risks taken; d) incomplete and false information on which the decision needs to be based; e) similar previous experiences; f)... However, deciding is always a situation in which there is never a "transparent" support, in which there is a percentage (greater or lesser) of luck, in which there is an important intervention of intuition. This is only acceptable because phenomena are always complex, not isolated and constantly changing.

\section{Managing Holes Versus Managing Matter}

The questions are unoccupied spaces (which, apparently, seek answers to fill them out), which, as in a kind of maze give way to more questions. However, this ambiguity is the most achieved way to satisfy those who are in search of uncertainty and the unknown. In the time of the "navigators and discoveries", ships went in search of new lands, of unknown worlds. But if they did find wonderful lands (on a trip that fulfilled their objectives fully) the tendency would not be to go back to where they were in the old days, but to take advantage of the better possibilities offered to them. Which was not, of course, the initial goal of the trip.

The different Stages of Knowledge Development do not Represent a Continuum, but Imply Ruptures in the Evolution of the Process

The information in its evolution gives rise to the ability to understand / explain, in view of the ability to choose and decide (see point 1 ). Desirably this process will lead to the production of a knowledge (which is not to know). A knowledge that, we intend, is not specific and local, but is generic and as universal as possible and that will lead (if we are lucky, ingenuity and digestion/reflection capacity) to the domain of a wisdom, which is still less material and more universal than the forms available in the previous phases (ground, for example, the five points with which we mark scarcities and plenty's at the beginning of this work).

In the face of these paradoxes, and the immensity of all the others with which we could illustrate the positions we have taken and defend here, we believe that we can affirm that a rupture (Kuhn again) is imposed, one that frees science from the shackles that drags from the past, a past, perhaps still recent (some would say current) in which we gain coherences and balances that free us from the need to permanently stumble on the cultures of the past (not those that are old but those that belong to the past and "passed the deadline"), the mentalities of the past (not those that are old but those that belong to the past and "passed the deadline"), the structures of the past (not those that are old but those that belong to the past and become "outdated"), the objectives and strategies of the past (not those that are old but those that belong to the past and become "outdated"). Huge challenges. What is good (or great?) for those who like reps, but bad for those who want stability (which is the reverse of movement).

Teaching / Education / Training has prepared us for integration into what exists, but not for the construction of futures, however desirable (and affirmed as desired) as they may be. Conflicts are increasingly imminent and evident. Let us hope that it is not decided and arbitrated by artificial intelligence (on the one hand and by natural stupidity on the other) or by a virus, (or both), that puts man and his social structures in a corner, because they consider them outdated (not because they are old but because they belong to the past and "passed the deadline".

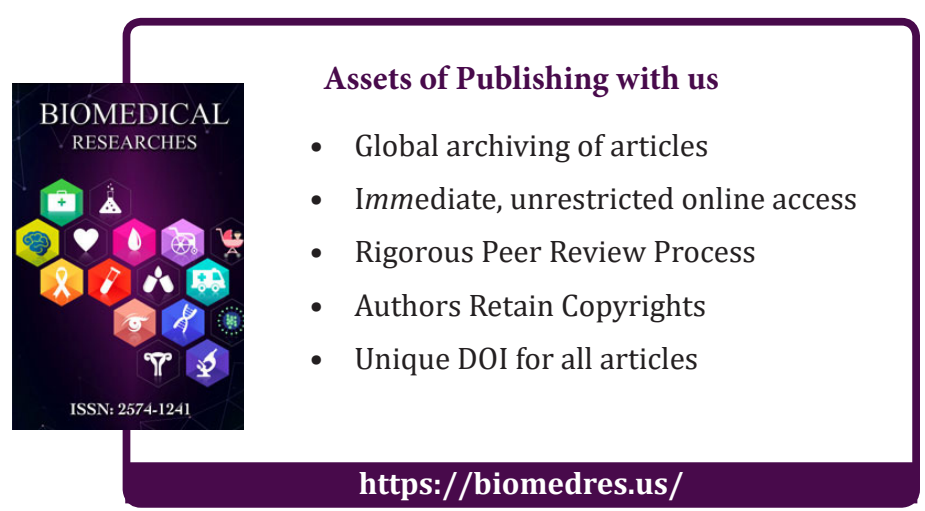

